\title{
Factors affecting Fontan length of stay: Results from the Single Ventricle Reconstruction trial
}

\author{
Chitra Ravishankar, MBBS, ${ }^{a}$ Eric Gerstenberger, MS, ${ }^{\mathrm{b}}$ Lynn A. Sleeper, ScD, ${ }^{\mathrm{b}}$ Andrew M. Atz, MD, ${ }^{\mathrm{c}}$ \\ Jeremy T. Affolter, MD, ${ }^{\mathrm{d}}$ Timothy J. Bradley, MBChB,${ }^{\mathrm{e}} \mathrm{J}$. William Gaynor, MD, ${ }^{\mathrm{f}}$ Bryan H. Goldstein, MD, ${ }^{\mathrm{g}}$ \\ Heather T. Henderson, MD, Jeffrey P. Jacobs, MD, ${ }^{\mathrm{i}}$ Alan B. Lewis, MD ${ }^{\mathrm{j}}$ Carolyn Dunbar-Masterson, RN, ${ }^{\mathrm{k}}$ \\ Shaji C. Menon, MD, ${ }^{1}$ Victoria L. Pemberton, RN, MS, ${ }^{\mathrm{m}}$ Christopher J. Petit, MD, ${ }^{\mathrm{n}}$ Nancy A. Pike, PhD, ${ }^{\circ}$ \\ Christian Pizarro, MD, ${ }^{\mathrm{p}}$ Kurt R. Schumacher, MD, ${ }^{\mathrm{q}}$ Ismee A. Williams, MD, MS, ${ }^{\mathrm{r}}$ and \\ Jane W. Newburger, MD, MPH, ${ }^{\mathrm{k}}$ for the Pediatric Heart Network Investigators
}

\begin{abstract}
Background: In the Single Ventricle Reconstruction trial, infants with hypoplastic left heart syndrome (HLHS) who received a right-ventricle-to-pulmonaryartery shunt (RVPAS) versus a modified Blalock-Taussig shunt (MBTS) had lower early postoperative mortality, but more complications at 14 months. We explored the effect of shunt type and other patient, medical, and surgical factors on postoperative length of stay (LOS) after the Fontan operation.
\end{abstract}

Methods: Fontan postoperative course was ascertained from medical record review. Cox proportional hazards modeling was used to identify factors associated with LOS.

Results: Of 327 subjects who underwent Fontan, 323 were analyzed (1 death, 1 biventricular repair, 2 with missing data). Median age and weight at Fontan were 2.8 years (interquartile range [IQR]: $2.3,3.4$ ) and $12.7 \mathrm{~kg}$ (IQR: 11.4, 14.1 ), respectively. Fontan type was extracardiac in $55 \%$ and lateral tunnel in $45 \% ; 87 \%$ were fenestrated. The RVPAS and MBTS subjects had similar LOS (median 11 days [IQR: 9, 18] vs 10 days [IQR: 9, 13]; $P=.23$ ). Independent risk factors for longer LOS were treatment center $(P<.01)$, LOS at stage II (hazard ratio [HR] 1.02 for each additional day; $P<.01$ ), and pre-Fontan complications (HR 1.03 for each additional complication; $P=.04$ ). Use of deep hypothermic circulatory arrest at Fontan (HR 0.64; $P=.02$ ) was independently associated with shorter LOS. When center was excluded from the model, preFontan complications and use of circulatory arrest were no longer significant; instead, older age at stage II (HR 1.08 for each additional month; $P=.01$ ) predicted longer LOS. In 254 subjects who had a pre-Fontan echocardiogram, at least moderate tricuspid regurgitation was independently associated with longer LOS,

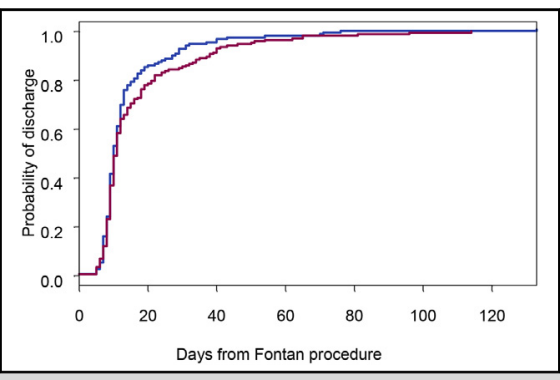

Kaplan-Meier curves comparing the time to hospital discharge after a Fontan operation for the 2 shunt groups (blue = modified Blalock-Taussig shunt; red = rightventricle-to-pulmonary-artery shunt) in transplant-free survivors (logrank $P=.24)$

\section{Central Message}

Longer length of stay after a Fontan procedure was associated with greater overall earlier medical complexity, and not with the shunt type used in a Norwood operation.

\section{Perspective}

In this follow-up analysis of the multicenter, prospective Single Ventricle Reconstruction trial cohort, survival after a Fontan operation was $99.7 \%$. Length of stay after the Fontan procedure was not associated with shunt type. Rather, global measures of earlier medical morbidity predicted longer LOS. Future studies are needed to assess the impact of early postoperative morbidity on late Fontan outcomes.

See Editorial Commentary page 676.

\footnotetext{
From the ${ }^{\text {a Department }}$ of Pediatrics, Children's Hospital of Philadelphia, Philadelphia, Pa; ${ }^{b}$ New England Research Institute, Watertown, Mass; ${ }^{c}$ Department of Pediatrics, Medical University of South Carolina, Charleston, SC; ${ }^{\mathrm{d} D e p a r t m e n t ~ o f ~}$ Critical Care Medicine, The Children's Hospital of Wisconsin, Milwaukee, Wis; ${ }^{\mathrm{e}}$ Department of Pediatrics, Hospital for Sick Children, Toronto, Ontario, Canada; ${ }^{\mathrm{f}}$ Division of Cardiothoracic Surgery, Children's Hospital of Philadelphia, Philadelphia, Pa; ${ }^{g}$ Department of Pediatrics, Cincinnati Children's Hospital Medical Center, Cincinnati, Ohio; hepartment of Pediatrics, Duke Medical Center, Durham,

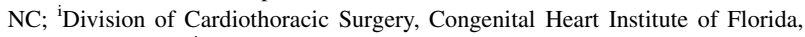
St Petersburg, Fla; ${ }^{\mathrm{j}}$ Department of Pediatrics, Children's Hospital of Los Angeles, Los Angeles, Calif; ${ }^{k}$ Department of Cardiology, Boston Children's Hospital, Boston, Mass; 'University of Utah, Department of Pediatrics, Salt Lake City, Utah; ${ }^{\mathrm{m}}$ National Heart, Lung, and Blood Institute, National Institutes of Health, Bethesda, Md; ${ }^{\mathrm{n}}$ Department of Pediatrics, Sibley Heart Center, Atlanta, Ga; ${ }^{\circ}$ Department of Nursing, University of California, Los Angeles, Los Angeles, Calif; ${ }^{\mathrm{P}}$ Division of Cardiothoracic Surgery, Alfred I. DuPont Hospital for Children,
}

Wilmington, Del; ${ }^{9}$ Department of Pediatrics, University of Michigan Health Center, Ann Arbor, Mich; and ${ }^{\mathrm{r}}$ Department of Pediatrics, Columbia University Medical Center, New York, NY.

This work was supported by grants HL068269, HL068270, HL068279, HL068281, HL068285, HL068288, HL068290, HL068292, and HL085057, from the National Heart, Lung, and Blood Institute (NHLBI). This work is solely the responsibility of the authors and does not necessarily represent the official views of NHLBI or the National Institutes of Health.

Received for publication April 29, 2015; revisions received Aug 20, 2015; accepted for publication Sept 4, 2015; available ahead of print Oct 28, 2015.

Address for reprints: Chitra Ravishankar, MBBS, Division of Cardiology, The Children's Hospital of Philadelphia, The Perelman School of Medicine, Philadelphia, PA 19104 (E-mail: ravishankar@email.chop.edu). $0022-5223 / \$ 36.00$

Copyright (C) 2016 by The American Association for Thoracic Surgery http://dx.doi.org/10.1016/j.jtcvs.2015.09.061 


\section{Abbreviations and Acronyms}

DHCA $=$ deep hypothermic circulatory arrest

HLHS = hypoplastic left heart syndrome

HR = hazard ratio

$\mathrm{IQR}=$ interquartile range

LOS = length of stay

MBTS $=$ modified Blalock-Taussig shunt

RVPAS = right-ventricle-to-pulmonary-artery shunt

SVR $=$ Single Ventricle Reconstruction trial

Scanning this QR code will take you appendix for this article.

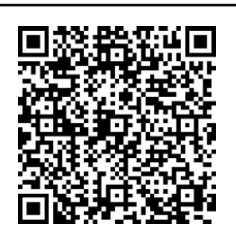

both with center (HR 1.72; $P<.01)$ and without center in the model (HR 1.49; $P=.02$ ).

Conclusions: In this multicenter prospective cohort of subjects with HLHS, Norwood shunt type was not associated with Fontan LOS. Rather, global measures of earlier medical complexity indicate greater likelihood of longer LOS after the Fontan operation. (J Thorac Cardiovasc Surg 2016;151:669-75)

Since its original description, the Fontan operation has been performed for various single-ventricle anomalies. ${ }^{1}$ The Fontan circuit relies on systemic venous pressure to propel blood through the lungs; it therefore depends on low pulmonary vascular resistance, which in turn is affected by such factors as ventricular function and pulmonary artery distortion. Few studies have explored the role of shunt type during the Norwood procedure for hypoplastic left heart syndrome (HLHS) in modulating these factors and affecting the Fontan postoperative course. ${ }^{2}$

The Single Ventricle Reconstruction (SVR) trial showed better transplant-free survival at 12 months in patients assigned to the right-ventricle-to-pulmonary-artery shunt (RVPAS), compared with the modified Blalock-Taussig shunt (MBTS), at the time of the Norwood operation for HLHS and other, related, single right-ventricular anomalies. ${ }^{3}$ However, subjects in the RVPAS group had more pulmonary artery stenosis, smaller pulmonary arteries, greater need for intervention, and more complications. These results raise concern, given that pulmonary artery stenosis has been associated with poor outcomes after the Fontan operation. ${ }^{4}$ In addition, the incision in the right ventricle that is required for placement of the RVPAS can lead to scar formation, which has the potential to diminish ventricular function and be a nidus for arrhythmia. ${ }^{5-7}$ In the SVR trial cohort, by the time of pre-Fontan echocardiography, survivors who had been randomized to the RVPAS, compared with the group who received the MBTS shunt, showed greater deterioration in right-ventricular ejection fraction. ${ }^{8,9}$

All transplant-free survivors of the SVR trial were eligible for enrollment in the SVR Extension study (SVR II), which compares clinical outcomes and right-ventricular performance through 6 years postrandomization. Annual medical histories were obtained when patients were between 2 and 6 years old, and data were collected during the Fontan procedure hospitalization. This well characterized cohort provides a unique opportunity to describe outcomes after the Fontan operation for HLHS, and related, single right-ventricular anomalies, and to determine factors that affect these outcomes from birth to the time of the Fontan procedure.

We hypothesized that subjects who received an RVPAS would have a longer postoperative length of stay (LOS) after undergoing the Fontan procedure, compared with those who received an MBTS. Postoperative LOS was specifically selected as the primary outcome for this analysis, owing to the anticipated low incidence of death, transplantation, or Fontan takedown in this cohort. In addition, we sought to determine the association of patient characteristics with medical and surgical factors relating to LOS after the Fontan operation.

\section{METHODS}

\section{Study Design and Data Collection}

In the Pediatric Heart Network's SVR trial, ${ }^{3}$ infants with HLHS and related, single right-ventricular anomalies, from 15 centers across North America, were randomized to receive the RVPAS or MBTS during the Norwood operation. Patients who had undergone the Fontan operation as of April 1, 2013 were eligible for inclusion in this analysis. The study was approved by the institutional review board or research ethics board at each participating center, and written informed consent was obtained from parents and/or guardians before enrollment in the trial.

The shunt that was in place at the end of the Norwood operation was used for the current analysis (as opposed to intention to treat). An annual medical history was obtained from transplant-free survivors, via phone interview with parents and/or guardians and review of medical records. Medical factors that were analyzed include the following: LOS, at both Norwood and stage II; the number of serious adverse events in the first 12 months, and the number of complications and interventional catheterizations in the time period from before the Norwood to the Fontan procedure.

Nonfatal serious adverse events that were recorded from the time of randomization in the SVR trial to age 12 months included acute shunt failure, cardiac arrest, use of extracorporeal membrane oxygenation support, unplanned cardiovascular reoperation, and necrotizing enterocolitis. Other potential risk factors, including patient characteristics, are listed in Appendix E1. Only those pre-Fontan echocardiograms that were performed at the study center were reviewed in a core laboratory, and pre-Fontan cardiac catheterization was performed per standard clinical practices at each center. The month in which the Fontan operation was performed was included as a potential seasonal predictor of LOS, with 2 categories: November through March (the peak time of year for viral 
respiratory illnesses to occur); and April through October. The course of patients' health after the Fontan operation was ascertained by review of medical records.

\section{Statistical Analysis}

Hospital LOS was defined as the number of days from the date of the Fontan operation (day 1) to the discharge date. Descriptive statistics are presented as mean $\pm \mathrm{SD}$, medians with interquartile range (IQR), and percentages, as appropriate. A univariate Cox proportional hazard regression model was used to identify associations of clinical factors with time to hospital discharge after the Fontan operation. The proportional hazard assumption was verified. Associations that were significant at $P \leq .20$ were considered for inclusion in the multivariable model.

Owing to the large amount of missing data, variables that were related to genetic assessment, pre-Fontan echocardiogram results and use of preFontan cardiac catheterization were initially excluded from modeling. A stepwise selection method was used, with multivariable Cox regression, to build 2 models that included or excluded, as a variable, the center where patients were treated. Based on these 2 models (ie, with vs without treatment center), the variables related to genetic assessment, pre-Fontan echocardiogram results, and use of pre-Fontan catheterization were added (each group separately), to determine their impact. All analyses were conducted using SAS, version 9.3 (SAS Institute, Inc, Cary, NC).

\section{RESULTS}

Among 549 SVR trial subjects who were randomized to the RVPAS or MBTS shunt for the Norwood operation, stage II was performed for $400(73 \%) ; 380(95 \%)$ of these patients had transplant-free survival to discharge. As of April 1, 2013, of these 380 patients, 21 had died, 10 had received transplants, 1 was listed for transplant, Fontan was planned in 11 and not planned in 6,3 were alive with unknown status, and 1 was lost to follow-up.

The Fontan operation was performed in 327 transplantfree survivors, from January 2007 to April 2013, of whom 323 constitute our analytic cohort. Of the remaining 4, 1 died, 2 had no available perioperative data, and 1 underwent biventricular repair during the Fontan hospitalization. The most common anatomic defect was HLHS, which was present in 285 patients $(88 \%)$. Five subjects had obstructed pulmonary venous return, and 2 had heterotaxy. The types of stage II procedures performed were as follows: bidirectional Glenn in 192 (59\%); hemi-Fontan in 84 $(26 \%)$; bilateral bidirectional Glenn in $34(11 \%)$; and other in $13(4 \%)$.

Nearly half of the subjects $(n=154)$ had undergone $\geq 1$ interventional catheterization before the Fontan operation. Interventions included balloon and/or stent angioplasty for coarctation in $66(20 \%)$, pulmonary artery balloon and/or stent angioplasty in $24(7 \%)$, and coil embolization of aortopulmonary collateral vessels in $56(17 \%)$. In the 254 patients who had a pre-Fontan echocardiogram that was evaluable for degree of tricuspid regurgitation, at least moderate tricuspid regurgitation was present in 45 $(18 \%)$. Cardiac catheterization in anticipation of the Fontan procedure was performed in $260(80 \%)$ patients. The mean systemic ventricular end-diastolic pressure and the transpulmonary gradient were $8.3 \pm 2.8$ and $3.4 \pm 2.9 \mathrm{~mm} \mathrm{Hg}$, respectively. Pulmonary artery abnormalities were documented on angiography in 76 of $260(29 \%)$ patients.

Baseline characteristics by shunt type, for those who underwent the Fontan procedure, indicate that the MBTS group had both a lower weight-for-age $\mathrm{z}$-score $(-0.9 \pm 1.1$ vs $-0.6 \pm 1.2 ; P=.03)$ and a lower heightfor-age $\mathrm{z}$-score $(-1.4 \pm 1.4$ vs $-1.0 \pm 1.5 ; P=.01)$ at 14 months, compared with those in the RVPAS group (Table 1). Serious adverse events in the first 12 months occurred more frequently in the MBTS group $(P=.03)$. However, those who received the MBTS had fewer cardiac catheterizations during the time period from birth to the Fontan procedure (n $[\mathrm{IQR}]):(0[0,1]$ vs $1[0,2]$; $P=.001)$. No other differences were found between the 2 groups.

\section{Fontan Procedure Characteristics}

Median age and weight at the time of the Fontan procedure were 2.8 years (IQR: $2.3,3.4$ ) and $12.7 \mathrm{~kg}$ (IQR: $11.4,14.1)$, respectively. The median weight-for-age $\mathrm{z}$-score and height-for-age $\mathrm{z}$-score were -0.5 (IQR: -1.3 to 0.1 ) and -1.1 ( -1.8 to -0.3$)$, respectively. The Fontan procedure type was extracardiac in $178(55 \%)$, and lateral tunnel in $145(45 \%)$ patients; $280(87 \%)$ underwent fenestration. Subjects undergoing the extracardiac procedure were older than those who underwent the lateral tunnel procedure $(3.2 \pm 0.8$ vs $2.5 \pm 0.7$ years; $P<.001)$. Deep hypothermic circulatory arrest (DHCA) was used for 93 $(29 \%)$ subjects. Concurrent procedures were performed at the time of the Fontan in $104(32 \%)$ subjects, with the 2 most common additional procedures being pulmonary arterioplasty for pulmonary artery stenosis (45 [14\%]), and atrioventricular valvuloplasty or repair in $29(9 \%)$ (Table 2).

\section{Postoperative Outcomes}

One patient died, and none required either Fontan takedown or transplantation before discharge. The median LOS for survivors was 10 days, and it was similar for the 2 shunt types (11 days [IQR: 9, 18] for RVPAS vs 10 days [IQR: 9,13$]$ for MBTS; $P=.23$ ) (Figure 1). Pleural drainage that persisted for $>7$ days occurred in $58(18 \%)$ patients. Other complications included arrhythmias in 50 $(15 \%)$, infections in $37(11 \%)$, and thromboembolic complications in $7(2 \%)$. Thirty-one $(10 \%)$ patients were readmitted within 30 days of discharge. None of these outcomes differed by shunt type.

\section{Factors Associated with Length of Stay}

Factors associated with longer LOS, by univariate analysis, are listed in Table 3. In the final multivariable model that included center (Table 4), independent risk factors for 
TABLE 1. Baseline characteristics of subjects, by shunt type $(n=323)$

\begin{tabular}{|c|c|c|c|c|c|}
\hline Characteristic & $\mathbf{n}$ & MBTS & $\mathbf{n}$ & RVPAS & $P$ value \\
\hline Gestational age (wk) & 150 & $38.4 \pm 1.3$ & 173 & $38.3 \pm 1.4$ & .77 \\
\hline Gestational age $<37$ wk & 150 & $10(7)$ & 173 & $14(8)$ & .63 \\
\hline Birth weight (kg) & 150 & $3.2 \pm 0.5$ & 173 & $3.1 \pm 0.5$ & .38 \\
\hline Birth weight $<2.5 \mathrm{~kg}$ & 150 & $18(12)$ & 173 & $13(8)$ & .17 \\
\hline Gender, male & 150 & $96(64)$ & 173 & $111(64)$ & .98 \\
\hline White race & 149 & $128(86)$ & 172 & $141(82)$ & .34 \\
\hline Hispanic race & 147 & $21(14)$ & 172 & $35(20)$ & .16 \\
\hline Socioeconomic score & 143 & $0.2 \pm 4.7$ & 169 & $0.6 \pm 5.6$ & .50 \\
\hline Below federal poverty level $(\%)$ & 143 & $8.4(3.2,13.3)$ & 169 & $8.3(3.9,17.2)$ & .19 \\
\hline Hypoplastic left heart syndrome & 150 & $131(87)$ & 173 & $154(89)$ & .64 \\
\hline Aortic atresia & 150 & $90(60)$ & 173 & $111(64)$ & .44 \\
\hline Genetic syndrome & 150 & & 173 & & .64 \\
\hline Yes & & $7(5)$ & & $5(3)$ & \\
\hline Unknown & & $28(19)$ & & $37(21)$ & \\
\hline No & & $115(77)$ & & $131(76)$ & \\
\hline Nonsyndromic abnormality & 150 & & 173 & & .77 \\
\hline Yes & & $32(21)$ & & $40(23)$ & \\
\hline Unknown & & $28(19)$ & & $36(21)$ & \\
\hline No & & $90(60)$ & & $97(56)$ & \\
\hline Center single-ventricle treatment volume & 150 & $27.5(18.9,32.2)$ & 173 & $27.5(17.1,32.2)$ & .20 \\
\hline Surgeon Norwood volume & 150 & $12.6(7.3,14.8)$ & 173 & $12.6(7.3,13.6)$ & .63 \\
\hline Age at stage II (mo) & 150 & $5.5 \pm 2.2$ & 173 & $5.2 \pm 1.6$ & .20 \\
\hline Length of stay at stage II (d) & 150 & $8(6,11)$ & 173 & $8(6,13)$ & .26 \\
\hline Weight for age $\mathrm{z}$-score, $14 \mathrm{mo}$ (WHO) & 139 & $-0.9 \pm 1.1$ & 163 & $-0.6 \pm 1.2$ & .03 \\
\hline Length for age z-score, 14 mo (WHO) & 139 & $-1.4 \pm 1.4$ & 163 & $-1.0 \pm 1.5$ & .01 \\
\hline Cardiac surgeries prior to Fontan & 150 & $1.0(0.0,1.0)$ & 173 & $1.0(0.0,1.0)$ & .47 \\
\hline Noncardiac surgeries prior to Fontan & 150 & $0.0(0.0,1.0)$ & 173 & $0.0(0.0,1.0)$ & .98 \\
\hline Complications prior to Fontan & 150 & $5.0(3.0,8.0)$ & 173 & $5.0(3.0,8.0)$ & .95 \\
\hline Serious adverse events (12 mo) & 150 & $0.0(0.0,1.0)$ & 173 & $0.0(0.0,0.0)$ & .03 \\
\hline Catheterization prior to Fontan & 150 & $0.0(0.0,1.0)$ & 173 & $1.0(0.0,2.0)$ & .001 \\
\hline$\geq$ Moderate tricuspid regurgitation on pre-Fontan echocardiogram & 119 & $20(17)$ & 136 & $25(18)$ & .87 \\
\hline Age at Fontan $(y)$ & 150 & $2.8 \pm 0.8$ & 173 & $2.9 \pm 0.8$ & .21 \\
\hline Weight for age z-score at Fontan & 116 & $-0.6 \pm 1.0$ & 142 & $-0.6 \pm 1.0$ & .79 \\
\hline Length for age z-score at Fontan & 116 & $-1.1 \pm 1.3$ & 141 & $-1.0 \pm 1.3$ & .59 \\
\hline
\end{tabular}

Values are $\mathrm{n}(\%)$, mean $\pm \mathrm{SD}$, or median (interquartile range), unless otherwise indicated. Socioeconomic score was assigned using a US Census-based score derived from 6 measures related to income, housing, and occupationally related features of the subject's census block tract at the time of randomization. MBTS, Modified Blalock-Taussig shunt; RVPAS, right-ventricle-to-pulmonary-artery shunt; $W H O$, World Health Organization.

longer LOS were as follows: treatment center $(P<.01)$; LOS at stage II (hazard ratio [HR] 1.02 for each additional day; $P<.01$ ); and pre-Fontan complications (HR 1.03 for each additional complication; $P=.04)$ Use of DHCA at the time of the Fontan procedure (HR 0.64; $P=.02$ ) was independently associated with shorter LOS. When the treatment center variable was excluded from the multivariable model, pre-Fontan complications and use of DHCA were no longer significant, and older age at stage II (HR 1.08 for each additional month; $P=.01$ ) entered the model and was independently associated with longer LOS.

In a subanalysis of 254 subjects in whom pre-Fontan echocardiograms were interpreted in an echocardiographic core laboratory (Table 5), presence of at least moderate tricuspid regurgitation was independently associated with longer LOS in multivariable models, both those that included treatment center (HR 1.72; $P<.01$ ) and those that excluded treatment center (HR 1.49; $P=.02$ ). Ventricular end-diastolic pressure and pulmonary artery abnormalities from pre-Fontan catheterization $(n=260)$ were each associated with longer LOS in univariate analysis, but not in the final multivariable model.

\section{DISCUSSION}

With advances in surgical techniques and perioperative care, the Fontan procedure surgery has low mortality in the current era. ${ }^{10-15}$ For patients with HLHS, and other, related, single right-ventricular anomalies, the Norwood operation performed with the RVPAS, compared with the MBTS, has been associated with a lower right-ventricular ejection fraction and more pulmonary artery distortion, 
TABLE 2. Procedures performed in 104 of 323 subjects, concurrently with the Fontan operation

\begin{tabular}{lr}
\hline \multicolumn{1}{c}{ Procedures } & $\mathbf{n}$ \\
\hline Pulmonary arterioplasty & 45 \\
Atrioventricular valvuloplasty or repair & 29 \\
Atrial septectomy & 14 \\
Revision of superior vena cava connection & 2 \\
Aortic arch repair & 5 \\
Semilunar valvuloplasty or repair & 6 \\
Placement of permanent pacing wires & 7 \\
Pacemaker insertion & 4 \\
Resection of endocardial fibroelastosis & 6 \\
Other procedures & 24 \\
\hline
\end{tabular}

potentially heightening the risk of adverse hemodynamics after the Fontan procedure.

In this follow-up analysis of the multicenter prospective SVR trial cohort, we found that transplant-free survival after the Fontan operation was $99.7 \%$, with a median LOS of 10 days. Contrary to our study hypothesis, morbidity after the Fontan procedure, as indicated by postoperative LOS, was not associated with type of Norwood shunt. We found that global measures of earlier medical complexity were associated with longer LOS after Fontan surgery, including LOS at stage II, pre-Fontan procedure complications, and presence of at least moderate tricuspid regurgitation. This study is the first multicenter analysis to explore risk factors for morbidity after the Fontan procedure in patients followed prospectively from birth.

Our data are concordant with a single-center study that demonstrated no difference in LOS at the time of the Fontan procedure, by Norwood shunt type. ${ }^{2}$ Our results compare favorably with those of other contemporary series that have reported mortality of $1 \%$ to $5 \%$, and LOS of 8 to 11 days after the Fontan procedure, for various singleventricle anomalies. ${ }^{10-15}$ The SVR trial cohort is

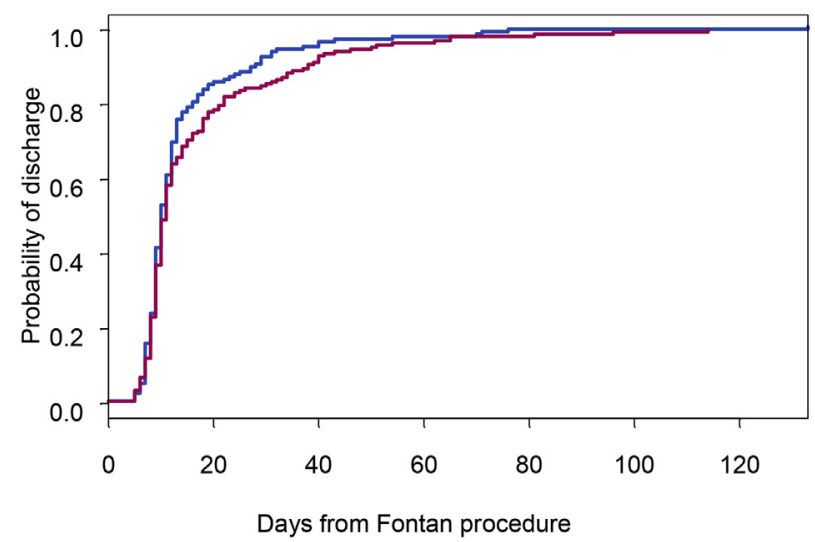

FIGURE 1. Kaplan-Meier curves comparing the time to hospital discharge after a Fontan operation for the 2 shunt groups (blue $=$ modified BlalockTaussig shunt; red $=$ right-ventricle-to-pulmonary-artery shunt) in transplant-free survivors (logrank $P=.24)$.
TABLE 3. List of potential predictors to include in the multivariable model $(P \leq .20$ in univariate analyses $)$

\begin{tabular}{lc}
\hline \multicolumn{1}{c}{ Predictor } & $\boldsymbol{P}$ value \\
\hline Center & .09 \\
Birth weight & .16 \\
Low birth weight $(<2.5 \mathrm{~kg})$ & .06 \\
Ever had an identifiable genetic syndrome & .07 \\
Ever had any nonsyndromic abnormalities & .13 \\
Socioeconomic score & .2 \\
Census tract: percentage below poverty line & .03 \\
Norwood length of stay & .09 \\
Age at stage II & .03 \\
Stage II length of stay & $<.001$ \\
Number of balloon angioplasties and/or stents for coarctation & .12 \\
Number of pulmonary artery balloons and/or stent angioplasties & .17 \\
Any pulmonary artery balloon and/or stent angioplasty & .17 \\
Number of noncardiac surgeries & .002 \\
Number of complications from pre-Norwood to Fontan & .006 \\
Number of serious adverse events (through 12 mo) & .04 \\
Any cardiopulmonary resuscitation & .15 \\
$\geq$ Moderate tricuspid regurgitation at pre-Fontan echo & .01 \\
Ventricular end-diastolic pressure & .11 \\
Pulmonary artery abnormalities & .08 \\
Weight at Fontan & .11 \\
Type of Fontan & .14 \\
Deep hypothermic circulatory arrest at Fontan & .18 \\
Other concurrent cardiac surgical procedures with Fontan & .20 \\
\hline
\end{tabular}

composed primarily of children who have HLHS, which is a risk factor for greater perioperative morbidity after the Fontan operation. In the largest report to date, of 771 consecutive patients undergoing the Fontan procedure, HLHS was present in 379 patients and was an independent predictor of longer LOS. ${ }^{15}$ In another large series that included 330 subjects who had HLHS, prolonged chest tube drainage ( $>14$ days), which is a surrogate marker for longer LOS, was found to occur more frequently in patients who had a morphologic right ventricle. ${ }^{12}$

Our finding of an association between LOS at stage II and LOS at the time of the Fontan procedure has not been reported previously, although this correlation is not unexpected. Both stage II and the Fontan operation result in passive pulmonary blood flow from the systemic veins and can be affected by factors related to the integrity of the pulmonary artery vasculature and the overall performance of the single ventricle. Older age of the patient at stage II was independently associated with longer LOS. We did not identify a threshold, for age at stage II, beyond which the LOS at the time of the Fontan procedure was likely to be longer. Possibly, this finding relates to a tendency for higher-risk candidates to undergo stage II procedures later in their course of illness.

Another new observation of this study is an association between longer Fontan LOS and more complications before the Fontan operation. The number of such complications is 
TABLE 4. Multivariable model for all 323 subjects, with versus without clinical center as a predictor

\begin{tabular}{|c|c|c|c|c|}
\hline & With center & & Without center & \\
\hline Variable & Hazard ratio & $P$ value & Hazard ratio & $P$ value \\
\hline Treatment center & & $<.01$ & & \\
\hline Each additional month of age at stage II & & & 1.08 & .01 \\
\hline Each additional day in hospital after stage II & 1.02 & $<.01$ & 1.02 & $<.01$ \\
\hline Each additional complication from birth to Fontan & 1.03 & .04 & & \\
\hline Deep hypothermic circulatory arrest at Fontan & 0.64 & .02 & & \\
\hline
\end{tabular}

likely a surrogate marker for overall medical morbidity. These include, but are not limited to, feeding difficulties, infections, heart failure, and frequent hospitalizations in the years preceding Fontan procedure completion.

In this study, a moderate or severe degree of tricuspid regurgitation was present in 49 subjects at the time of a pre-Fontan procedure echocardiogram and was a risk factor for longer LOS. Tricuspid valve repair or valvuloplasty $(\mathrm{n}=29)$ was the second most common concomitant procedure that was done at the time of the Fontan procedure in SVR trial subjects. The presence of substantial tricuspid regurgitation remains a risk factor for worse outcomes through all stages of palliation. ${ }^{16,17}$

Atrioventricular valve regurgitation has been reported to be a risk factor for mortality and morbidity after the Fontan operation, in both short- and long-term follow-up. ${ }^{15,17}$ Indeed, the association of concurrent repair of the atrioventricular valve with greater mortality and morbidity after the Fontan procedure ${ }^{15}$ likely reflects the higher risk among those who require such repair, ie, so-called confounding by indication. Tricuspid regurgitation increases the volume load of the single right ventricle and can increase the ventricular end-diastolic pressure and pulmonary artery pressure, in turn contributing to pleural effusions in the immediate postoperative period.

The use of DHCA at the time of the Fontan procedure was independently associated with shorter LOS, in models allowing for treatment center to be a predictor. This finding may be explained by a reduced incidence of systemic inflammatory response syndrome provoked by DHCA. ${ }^{18,19}$ Other intraoperative factors, such as longer cardiopulmonary bypass time, ${ }^{20}$ and extracardiac Fontan procedure, ${ }^{21}$ have been associated with longer LOS in other studies, whereas fenestration and use of modified ultrafiltration have been found to be associated with shorter $\operatorname{LOS} .^{15}$ In our study, none of these factors predicted LOS; and no pre-Fontan procedure catheterization variables or catheter-based interventions were associated with LOS. Contrary to previous reports, performance of the Fontan operation from November through May (the peak season for viral respiratory infections) was not associated with a longer LOS. ${ }^{22,23}$

Our study has several limitations. We found that clinical center was a strong independent predictor of LOS after the Fontan procedure. Treatment center has been associated with outcomes in other analyses of the SVR trial, and practice pattern variation among participating sites was considerable. ${ }^{24}$ Our study design did not allow for us to determine which practice differences among centers were causal. Core-laboratory interpretation of pre-Fontan procedure echocardiograms was performed for $79 \%$ subjects, ie, only those echocardiograms performed at the study center, which limited the number available for analysis. Similarly incomplete data for other risk factors, such as weight of the patient at the time of the Fontan procedure, limited our ability to evaluate their impact on LOS.

Pre-Fontan procedure cardiac catheterization was performed in $80 \%$ of subjects, but pulmonary vascular resistance was not included in the prospective data collection. We did not collect data on total number of days of chest tube drainage after the Fontan procedure. Finally, the presence of a fenestration has been linked to shorter LOS after the Fontan procedure; because most subjects $(87 \%)$ in our analytic cohort had a fenestration created at the time of Fontan surgery, we could not assess the effect of fenestration on Fontan LOS.

In conclusion, in this large, prospective, multicenter trial of subjects who had HLHS and related, single rightventricular anomalies, early transplant-free survival after the Fontan operation was nearly $100 \%$. Despite concerns about the effect of the RVPAS on right-ventricular function

TABLE 5. Multivariable model in subjects with pre-Fontan echocardiogram $(n=254)$, with versus without treatment center as a predictor

\begin{tabular}{|c|c|c|c|c|}
\hline & With center & & Without center & \\
\hline Variable & Hazard ratio & $P$ value & Hazard ratio & $P$ value \\
\hline Treatment center & & $<.01$ & & \\
\hline Each additional month of age at stage II & & & 1.09 & .02 \\
\hline Each additional day in hospital after stage II & 1.02 & .03 & 1.02 & $<.01$ \\
\hline At least moderate tricuspid regurgitation on pre-Fontan echocardiogram & 1.72 & $<.01$ & 1.49 & .02 \\
\hline
\end{tabular}


and pulmonary artery architecture, Norwood shunt type did not affect LOS. Future studies are needed to assess whether morbidity in the early period after the Fontan procedure is correlated with late Fontan morbidities.

\section{Conflict of Interest Statement}

Authors have nothing to disclose with regard to commercial support.

\section{References}

1. Fontan F, Baudet E. Surgical repair of tricuspid atresia. Thorax. 1971;26:240-8.

2. Ballweg JA, Dominguez TE, Ravishankar C, Gaynor JW, Nicolson SC, Spray TL, et al. A contemporary comparison of the effect of shunt type in hypoplastic left heart syndrome on the hemodynamics and outcome at Fontan completion. J Thorac Cardiovasc Surg. 2010;140:537-44

3. Ohye RG, Sleeper LA, Mahony L, Newburger JW, Pearson GD, Lu M, et al; Pediatric Heart Network Investigators. Comparison of shunt types in the Norwood procedure for single-ventricle lesions. N Engl J Med. 2010;362:1980-92.

4. Gentles TL, Mayer JE Jr, Gauvreau K, Newburger JW, Lock JE, Kupferschmid JP, et al. Fontan operation in five hundred consecutive patients: factors influencing early and late outcome. J Thorac Cardiovasc Surg. 1997; 114:376-91.

5. Tanoue Y, Kado H, Shiokawa Y, Fusazaki N, Ishikawa S. Midterm ventricular performance after Norwood procedure with right ventricular-pulmonary artery conduit. Ann Thorac Surg. 2004;78:1965-71; discussion 1971.

6. Menon SC, Erickson LK, McFadden M, Miller DV. Effect of ventriculotomy on right-ventricular remodeling in hypoplastic left heart syndrome: a histopathology and echocardiography correlation study. Pediatr Cardiol. 2013; 34:354-63.

7. Padalino MA, Castellani C, Toffoli S, Della Barbera M, Milanesi O, Thiene G, et al. Pathological changes and myocardial remodelling related to the mode of shunting following surgical palliation for hypoplastic left heart syndrome. Cardiol Young. 2008;18:415-22.

8. Frommelt PC, Gerstenberger E, Cnota JF, Cohen MC, Gorentz J, Hill KD, et al; Pediatric Heart Network Investigators. Impact of initial shunt type on cardiac size and function in children with single right ventricle anomalies before the Fontan procedure: single ventricle reconstruction extension trial. J Am Coll Cardiol. 2014;64:2026-35

9. Newburger JW, Sleeper LA, Frommelt PC, Pearson GD, Mahle WT, Chen S, et al; Pediatric Heart Network Investigators. Transplantation-free survival and interventions at 3 years in the single ventricle reconstruction trial. Circulation. 2014;129:2013-20.

10. Petrossian E, Reddy VM, Collins KK, Culbertson CB, MacDonald MJ, Lamberti JJ, et al. The extracardiac conduit Fontan operation using minimal approach extracorporeal circulation: early and midterm outcomes. J Thorac Cardiovasc Surg. 2006;132:1054-63.
11. Tweddell JS, Nersesian M, Mussatto KA, Nugent M, Simpson P, Mitchell ME, et al. Fontan palliation in the modern era: factors impacting mortality and morbidity. Ann Thorac Surg. 2009;88:1291-9.

12. Hirsch JC, Goldberg C, Bove EL, Salehian S, Lee T, Ohye RG, et al. Fontan oper ation in the current era: a 15-year single institution experience. Ann Surg. 2008; 248:402-10.

13. Salvin JW, Scheurer MA, Laussen PC, Mayer JE Jr, Del Nido PJ, Pigula FA, et al Factors associated with prolonged recovery after the Fontan operation. Circulation. 2008;118:S171-6.

14. O'Brien JE Jr, Marshall JA, Young AR, Handley KM, Lofland GK. The nonfenestrated extracardiac Fontan procedure: a cohort of 145 patients. Ann Thorac Surg. 2010;89:1815-20.

15. Rogers LS, Glatz AC, Ravishankar C, Spray TL, Nicolson SC, Rychik R, et al. 18 years of the Fontan operation at a single institution. J Am Coll Cardiol. 2012;60: 1018-25.

16. Ghanayem NS, Allen KR, Tabbutt S, Atz AM, Clabby ML, Cooper DS, et al Pediatric Heart Network Investigators. Interstage mortality after the Norwood procedure: results of the multicenter Single Ventricle Reconstruction trial J Thorac Cardiovasc Surg. 2012;144:896-906.

17. Friedman KG, Salvin JW, Wypij D, Gurmu Y, Bacha EA, Brown DW, et al. Risk factors for failed staged palliation after bidirectional Glenn in infants who have undergone stage one palliation. Eur J Cardiothorac Surg. 2011;40:1000-6.

18. Schultz JM, Karamlou T, Swanson J, Shen I, Ungerleider RM. Hypothermic lowflow cardiopulmonary bypass impairs pulmonary and right ventricular function more than circulatory arrest. Ann Thorac Surg. 2006;81:474-80.

19. Tassani P, Barankay A, Haas F, Paek SU, Heilmaier M, Hess J, et al. Cardiac surgery with deep hypothermic circulatory arrest produces less systemic inflammatory response than low-flow cardiopulmonary bypass in newborns. J Thorac Cardiovasc Surg. 2002;123:648-54.

20. Gupta A, Daggett C, Behera S, Ferraro M, Wells W, Starnes V. Risk factors for persistent pleural effusions after the extracardiac Fontan procedure. J Thorac Cardiovasc Surg. 2004;127:1664-9.

21. Stewart RD, Pasquali SK, Jacobs JP, Benjamin DK, Jaggers J, Cheng J, et al. Contemporary Fontan operation: association between early outcome and type of cavopulmonary connection. Ann Thorac Surg. 2012;93:1254-60; discussion 1261.

22. Fedderly RT, Whitstone BN, Frisbee SJ, Tweddell JS, Litwin SB. Factors related to pleural effusions after Fontan procedure in the era of fenestration. Circulation. 2001; 18:148-51.

23. Salam S, Dominguez T, Tsang V, Giardini A. Longer hospital stay after Fontan completion in the November to March period. Eur J Cardiothorac Surg. 2015; 47:262-8.

24. Pasquali SK, Ohye RG, Lu M, Kaltman J, Caldarone CA, Pizarro C, et al; Pediatric Heart Network Investigators. Variation in perioperative care across centers for infants undergoing the Norwood procedure. J Thorac Cardiovasc Surg. 2012; 144:915-21

Key Words: Fontan, hypoplastic left heart syndrome, Single Ventricle Reconstruction trial 


\section{APPENDIX E1}

\section{List of Candidate Predictors}

\section{General}

- Treatment center

- Socioeconomic score

- Gender

- Race/ethnicity

- Anatomy

Hypoplastic left heart syndrome

Unbalanced atrioventricular canal defect

Other

- Heterotaxy (yes or no)

- Obstructed pulmonary veins defined by the use of postnatal intervention, including balloon septostomy, open atrial septectomy, or urgent Norwood procedure (yes or no)

- Gestational age

- Gestational age $<37$ weeks (yes or no)

- Birth weight

- Birth weight $<2500 \mathrm{~g}$ (yes or no)

- Diagnosed with genetic syndrome or nonsyndromic abnormalities (yes or no)

\section{Norwood Hospitalization}

- Shunt in place at the end of the Norwood procedure

- Length of hospital stay

\section{Stage II}

- Age at stage II

- Length of hospital stay

\section{Number of Serious Adverse Events (Through Age 12 Months)}

\section{From pre-Norwood to Fontan}

- Number of cardiac surgeries

- Number of noncardiac surgeries
- Number of complications

- Cardiopulmonary resuscitation (yes or no)

- Extracorporeal membrane oxygenation (yes or no)

- Number of interventional catheterizations

- Balloon angioplasty/stent for coarctation (yes or no, number)

- Pulmonary artery balloon/stent angioplasty (yes or no, number)

- Coil embolization of aortopulmonary collateral vessels (yes or no, number)

\section{Pre-Fontan}

- Echocardiogram

Ventricular function

Atrioventricular valve regurgitation

- Catheterization

Pre-Fontan catheterization performed (yes or no)

Right ventricular end-diastolic pressure

Mean pulmonary artery pressure

Mean atrial pressure

Transpulmonary gradient

Pulmonary artery abnormalities

\section{Fontan}

- Month of Fontan: November to March or April to October

- Age at Fontan

- Weight at Fontan

- Type of Fontan (extracardiac or lateral tunnel)

- Fenestration (yes or no)

- Total bypass time

- Total aortic crossclamp time

- Deep hypothermic circulatory arrest (yes or no)

- Other cardiac surgical procedures concurrent with Fontan (yes or no) 